\title{
Effectiveness of Mass Drug Administration on Neglected Tropical Diseases in Schoolchildren in Zanzibar, Tanzania
}

\author{
Ju Yeong Kim ${ }^{1,2, \dagger}$, Seobo Sim ${ }^{3, \dagger}$, Eun Joo Chung ${ }^{1}$, Han-Jong Rim4, Jong-Yil Chaj ${ }^{5,6}$, Duk-Young Min \\ Keeseon S. Eom ${ }^{8}$, Khalfan A. Mohammed ${ }^{9}$, Iddi S. Khamis ${ }^{9}$, Tai-Soon Yong ${ }^{1, *}$
}

\begin{abstract}
${ }^{1}$ Department of Environmental Medical Biology, Institute of Tropical Medicine and Arthropods of Medical Importance Resource Bank, Yonsei University College of Medicine, Seoul 03722, Korea; ${ }^{2 B}$ rain Korea 21 PLUS Project for Medical Science, Yonsei University College of Medicine, Seoul 03722, Korea; ${ }^{3}$ Department of Environmental and Tropical Medicine and International Healthcare Research Institute, Konkuk University School of Medicine, Seoul 05029, Korea; ${ }^{4}$ Department of Parasitology, College of Medicine, Korea University, Seoul 02841, Korea; ${ }^{5}$ Department of Tropical Medicine and Parasitology, Seoul National University College of Medicine, Seoul 03080, Korea; ${ }^{6}$ Institute of Parasitic Diseases, Korea Association of Health Promotion, Seoul 07649, Korea; ${ }^{7}$ Department of Microbiology and Immunology, Eulji University College of Medicine, Daejeon 34824, Korea; ${ }^{8}$ Department of Parasitology and Medical Research Institute, Parasite Resource Bank, Chungbuk National University School of Medicine, Cheongju 28644, Korea; 9 Integrated Helminth Control Program, Ministry of Health and Social Welfare, Zanzibar, Tanzania
\end{abstract}

\begin{abstract}
Soil-transmitted helminths and Schistosoma haematobium affect more than 3 billion people globally and mainly occur in sub-Saharan Africa. The present study assessed the overall infection status of a 1716-student cohort of schoolchildren in Zanzibar and applied mass drug administration (MDA) to the cohort from 2007 to 2009. Schools in Pemba, Zanzibar, had a much higher prevalence of soil-transmitted helminth infections than those in Unguja, and the Chaani, Ghana, and Machui schools of Unguja exhibited high S. haematobium infection rates. The MDA program only partially controlled parasite infections, owing to high rates of re-infection. The infection rate of $S$. haematobium across all 10 schools, for example, was only reduced by $1.8 \%$, and even this change not significant, even though the S. haematobium infection rates of the Chaani and Mzambarauni schools were significantly reduced from 64.4 and 23.4\%, respectively, at the first screening, to 7.3 and $2.3 \%$ at the last screening. The overall infection rate of Ascaris lumbricoides was reduced from $36.0 \%$ at the first screening to $22.6 \%$ at the last screening. However, the infection rates for both Trichuris trichiura and hookworm were generally unaffected by MDA. In the future, parasite control programs should involve strategically designed MDA schedules and holistic intervention (e.g., sanitation improvement, hygiene behavior changes, and control of intermediated hosts).
\end{abstract}

Key words: Schistosoma haematobium, soil-transmitted helminth, neglected tropical disease, mass drug administration, Zanzibar

\section{INTRODUCTION}

Infections by soil-transmitted helminths (STHs: Ascaris lumbricoides, Trichuris trichiura, and hookworm) and schistosomiasis, which is caused by Schistosoma haematobium, are considered neglected tropical diseases [1] and have been reported to affect more than 3 billion people globally, although mainly in sub-Saharan Africa [2]. These neglected tropical diseases are strongly associated with poverty [3] and contribute to anemia, impaired cognitive development, and the retardation of child-

\footnotetext{
- Received 26 July 2019, revised 25 November 2019, accepted 16 February 2020.

*Corresponding author (tsyong212@yuhs.ac)

†These authors contributed equally to this work.

(C) 2020, Korean Society for Parasitology and Tropical Medicine

This is an Open Access article distributed under the terms of the Creative Commons Attribution Non-Commercial License (https://creativecommons.org/licenses/by-nc/4.0) which permits unrestricted non-commercial use, distribution, and reproduction in any medium, provided the original work is properly cited.
}

hood growth, thereby preventing children from completing academic courses and sustaining their poverty $[4,5]$. In addition, $S$. haematobium infection is a risk factor for bladder cancer [6]. Therefore, the World Health Organization has developed a plan for eliminating STH and schistosome infections by 2020 [7], through the mass drug administration (MDA) to at-risk populations, including children [8].

The March 2009 report of the Ministry of Health of Zanzibar reported that an estimated one million people ( $82 \%$ of total population) throughout Zanzibar (Unguja and Pemba) are infected with S. haematobium and STHs, and although STH infections have been decreasing in Unguja, they are still problematic in Pemba [9-11]. Furthermore, urinary schistosomiasis has long been known to occur in Zanzibar and is known to affect school children [12-16]. The disease is locally transmitted by the freshwater snail Bulinus globosus, which establishes an 
endemic area of $S$. haematobium infection [13], since humans are infected by $S$. haematobium when they interact with water inhabited by B. globosus.

Accordingly, the purpose of the present study was to survey the parasitic infections rates of children in Zanzibar, to reduce overall parasitic infections using MDA, and to assess the association between parasitic infections and hemoglobin concentration or growth. A cohort of 1,716 children from 10 primary schools in Zanzibar (6 in Unguja and 4 in Pemba) were enrolled for 3 years' follow-up from 2007 to 2009. The parasitic infection status (Ascaris lumbricoides, Trichuris trichiura, hook- worm, and S. haematobium), body weight, and hemoglobin concentration of the children were assessed, and MDA was conducted.

\section{MATERIALS AND METHODS}

\section{Ethical considerations}

The present study was approved by the Ministry of Health and Social Welfare and Ministry of Education and Vocational Training, Zanzibar, under the agreement of the 'Korea-Zanzibar collaborative project on health promotion through para-

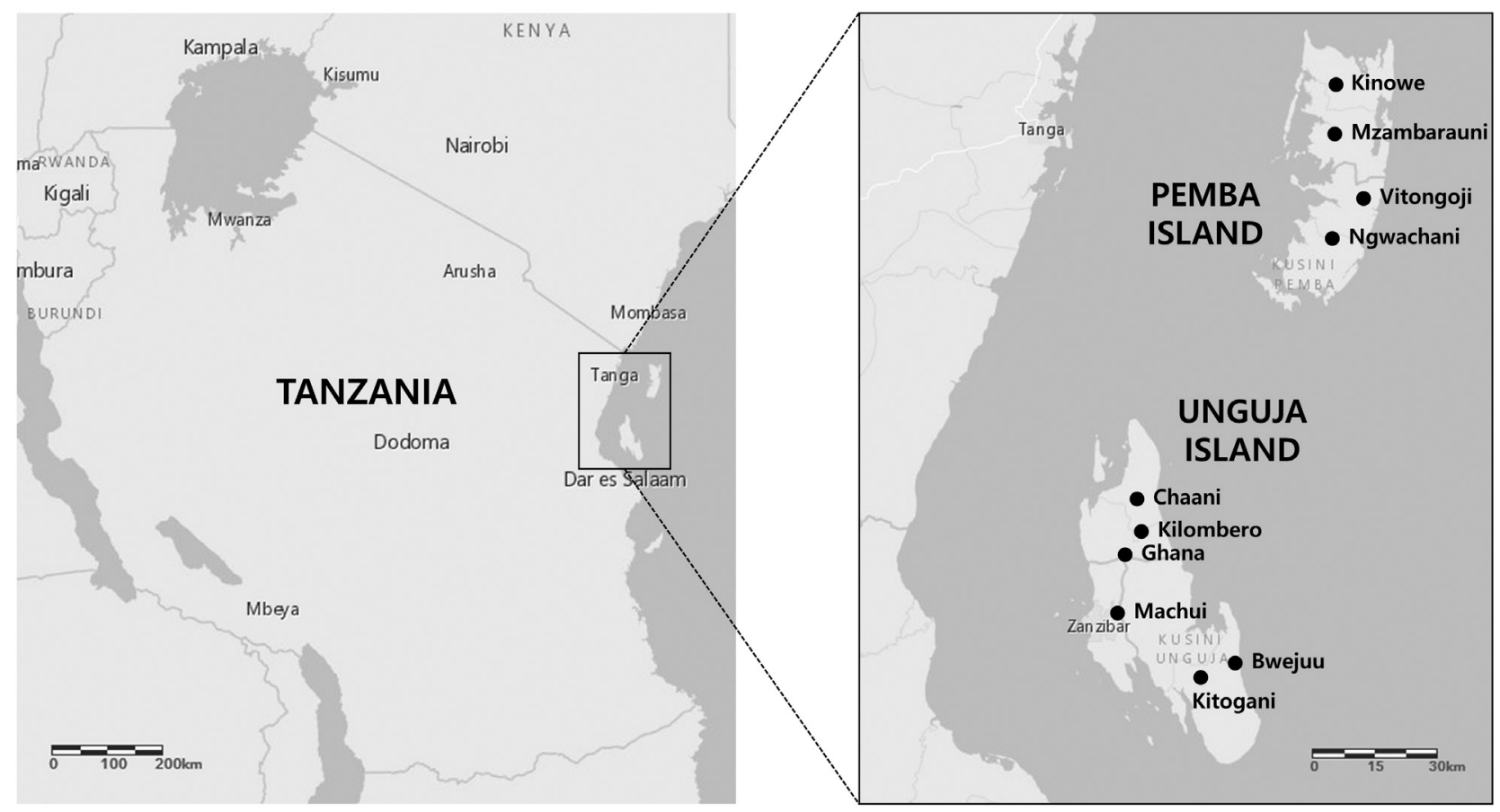

Fig. 1. Locations of the 10 primary schools included in the present study.

Table 1. Parasite screening of 1,716 schoolchildren in Zanzibar, Tanzania

\begin{tabular}{|c|c|c|c|c|c|}
\hline \multirow{2}{*}{ Island } & \multirow{2}{*}{ School } & 1st visit (Feb 2007) & 2nd visit (Jul 2007) & 3rd visit (Feb 2008) & 4th visit (Feb 2009) \\
\hline & & No. screened (male, female) & No. screened (male, female) & No. screened (male, female) & No. screened (male, female) \\
\hline \multirow[t]{6}{*}{ Unguja } & Chaani & $177(89,88)$ & & $103(46,57)$ & $109(34,75)$ \\
\hline & Kilombero & $149(67,82)$ & & $119(52,67)$ & $114(45,69)$ \\
\hline & Ghana & $167(80,87)$ & & $123(55,68)$ & $111(47,64)$ \\
\hline & Machui & $174(89,85)$ & & $138(67,71)$ & $107(45,62)$ \\
\hline & Kitogani & & $122(67,55)$ & $86(44,42)$ & $89(45,44)$ \\
\hline & Bwejuu & & $172(74,98)$ & $133(53,80)$ & $128(48,80)$ \\
\hline \multirow[t]{4}{*}{ Pemba } & Mzambarauni & & $202(95,107)$ & & $135(53,82)$ \\
\hline & Kinowe & & $187(73,114)$ & & $116(37,79)$ \\
\hline & Vitongoji & & $174(84,90)$ & & 103 (data lost) \\
\hline & Ngwachani & & $192(87,105)$ & & $129(56,73)$ \\
\hline Total & & $667(325,342)$ & $1,049(480,569)$ & $702(317,385)$ & 1,141 \\
\hline
\end{tabular}


site control among school children in Zanzibar (2007-2009)'. All participants provided written informed consent before participating in the study.

\section{Study area and population}

Zanzibar, which is a semi-autonomous archipelago in Tanzania, is located approximately $35 \mathrm{~km}$ from the coast of the mainland and possesses a population of $\sim 1.3$ million. The present study enrolled a total 1,716 children from 10 primary schools (Fig. 1), and the cohort was followed for 3 years (from 2007 to 2009; Table 1) to record the parasitic infection status, weight, and hemoglobin concentration of the children.

\section{Parasite examination}

Stool and urine samples were obtained from the enrolled children and processed in the laboratory within $24 \mathrm{hr}$. The stool samples were examined in duplicate for the presence of STH ova using the Kato-Katz thick smear technique, which included a sieve and calibrated template of $41.7 \mathrm{mg}$, and eggs were counted in the entire cellophane film. Meanwhile, the urine samples, which were processed using the urine filtration technique and polycarbonate membrane filters, were examined for the presence of $S$. haematobium eggs.

Hemoglobin concentrations were measured using a Hemocue (HemoCue AB, Ängelholm, Sweden). Regardless of gender, hemoglobin levels of $<11.5 \mathrm{~g} / \mathrm{dl}$ were interpreted as indicative of anemia, as specified by the World Health Organization for 6- to 12-year-old children.

\section{Anti-parasite treatment}

MDA on STH and schistosomiasis was conducted annually from 2007 to 2009 in the schools in Zanzibar (Table 1). At every visit, all the enrolled children were treated with a single dose of albendazole $(400 \mathrm{mg}$ ) to treat STH infection. The same children were also treated for schistosomiasis. Children who were diagnosed with schistosomiasis, using urine microscopy or owing to the presence of hematuria, were treated with praziquantel $(40 \mathrm{mg} / \mathrm{kg})$. Only 1,141 of the 1,716 enrolled children were examined during the last visit in February 2009.

\section{Statistical analysis}

The chi-square test, Fisher's exact test, one-way analysis of variance (ANOVA) with Bonferroni correction, and multiple logistic regression were performed using R 3.5.3 (http://www.Rproject.org), and $P$-values of $<0.05$ were considered significant.
The heatmap analysis and Principal Component Analysis (PCA) were performed using the Vegan package 2.5-4 in R 3.5.3.

\section{RESULTS}

The rate of $S$. haematobium infection was not effectively reduced by MDA. Indeed, even though the infection rates of the Chaani and Mzambarauni schools were reduced by 55.1 and $21.1 \%$ (Table 2), respectively, the overall reduction across all 10 schools was only $1.8 \%$, and even that small reduction was not statistically significant $(P=0.1751)$. Furthermore, the $S$. haematobium infection rates of several schools (Machui, Ghana, and Vitongoji) actually exhibited significant increases (Table 2).

In addition, of the 3 STHs, MDA was only effective against $A$. lumbricoides. The rate of A. lumbricoides infection across all schools was $36.0 \%$ at the beginning of the project and was reduced to $22.6 \%$ by the last screening $(P<0.0001)$. Therefore, MDA significantly reduced the rate of $A$. lumbricoides infection, especially in the 4 schools in Pemba. MDA also has significant effects (positive and negative) on the T. trichiura and hookworm infection rates of several individual schools (Table 2); however, there were no significant effects on the overall $T$. trichiura and hookworm infection rates across all 10 schools.

Heatmap analysis clustered schools with similar infections patterns for 4 parasites and visualized this data (Fig. 2). The Pemba schools were grouped together, based on their similarly high rates of STH infection, which were higher than those observed in the Unguja schools. Meanwhile, among the Unguja schools, the Chaani, Ghana, and Machui schools were grouped together, based on similarly high rates of $S$. haematobium infection, whereas the Kitogani and Bwejuu schools were grouped together, based on similarly low rates of S. haematobium infection. The Kilombero school exhibited higher STH infection rates than the other Unguja schools.

The 4 parasitic infection rates in each elementary school were expressed in a 2-dimensional graph using PCA. The PCA projected high-dimensional data (4 dimensions in this study) in 2 dimensions, resulting in 2 axes (PC1 and PC2) that make the largest distance between each point (this best describes the factors). The schools that had similar parasitic infection patterns were closely located in the graph (Fig. 3). The PCA results indicated that STH infections were more prevalent from the origin to the right and S. haematobium infection was more prevalent from the origin upwards. The vectors STH and $S$. 
Table 2. Parasitic infection rates of schoolchildren in 2007-2009

\begin{tabular}{|c|c|c|c|c|c|c|c|c|c|}
\hline \multicolumn{10}{|c|}{ Schistosoma haematobium infection } \\
\hline \multirow{2}{*}{ School } & \multicolumn{2}{|c|}{ 1st visit (Feb 2007) } & \multicolumn{2}{|c|}{ 2nd visit (Jul 2007) } & \multicolumn{2}{|c|}{ 3rd visit (Feb 2008) } & \multicolumn{2}{|c|}{ 4th visit (Feb 2009) } & \multirow{2}{*}{$\begin{array}{l}\text { Difference between 1st and } \\
\text { 4th screening ( } P \text {-value) }\end{array}$} \\
\hline & Screened & Cases (\%) & Screened & Cases (\%) & Screened & Cases (\%) & Screened & Cases (\%) & \\
\hline Chaani & 125 & $78(62.4)$ & & & 88 & $11(12.5)$ & 109 & $8(7.3)$ & $-55.1 \%(<0.0001)$ \\
\hline Kilombero & 126 & $11(8.7)$ & & & 118 & $3(2.5)$ & 111 & $5(4.5)$ & $-4.2 \%(0.1957)$ \\
\hline Ghana & 166 & $23(13.8)$ & & & 119 & $21(17.6)$ & 110 & $36(32.7)$ & $18.9 \%(0.0002)$ \\
\hline Machui & 168 & $14(8.3)$ & & & 136 & $56(41.2)$ & 107 & $35(32.7)$ & $24.4 \%(<0.0001)$ \\
\hline Kitogani & & & 122 & $5(4.1)$ & 86 & $0(0.0)$ & 88 & $2(2.3)$ & $-1.8 \%(0.4671)$ \\
\hline Bwejuu & & & 167 & $3(1.8)$ & 133 & $2(1.5)$ & 128 & $1(0.8)$ & $-1 \%(0.4549)$ \\
\hline Mzambarauni & & & 171 & $41(23.4)$ & & & 132 & $3(2.3)$ & $-21.1 \%(<0.0001)$ \\
\hline Kinowe & & & 186 & $5(2.7)$ & & & 113 & $4(3.5)$ & $0.8 \%(0.6760)$ \\
\hline Vitongoji & & & 173 & $22(12.7)$ & & & 103 & $26(25.2)$ & $12.5 \%(0.0079)$ \\
\hline Ngwachani & & & 185 & $19(10.3)$ & & & 129 & 17 (13.2) & $2.9 \%(0.4262)$ \\
\hline Total & & reened (Feb & and Jul 200 & 7): 1,589; Ca & ses: 221 (13 & 3.9) & 1,130 & $137(12.1)$ & $-1.8 \%(0.1751)$ \\
\hline \multicolumn{10}{|c|}{ Ascaris lumbricoides infection } \\
\hline \multirow{2}{*}{ School } & \multicolumn{2}{|c|}{ 1st visit (Feb 2007) } & \multicolumn{2}{|c|}{ 2nd visit (Jul 2007) } & \multicolumn{2}{|c|}{ 3rd visit (Feb 2008) } & \multicolumn{2}{|c|}{ 4th visit (Feb 2009) } & \multirow{2}{*}{$\begin{array}{l}\text { Difference between 1st and } \\
\text { 4th screening ( } P \text {-value) }\end{array}$} \\
\hline & Screened & Cases (\%) & Screened & Cases (\%) & Screened & Cases (\%) & Screened & Cases (\%) & \\
\hline Chaani & 129 & $3(2.3)$ & & & 61 & $10(16.4)$ & 43 & $3(7.0)$ & $4.7 \%(0.1500)$ \\
\hline Kilombero & 102 & $31(30.4)$ & & & 62 & $9(14.5)$ & 114 & $7(6.1)$ & $-24.3 \%(<0.0001)$ \\
\hline Ghana & 119 & $6(5.0)$ & & & 88 & $8(9.1)$ & 60 & $2(3.3)$ & $-1.7 \%(0.6015)$ \\
\hline Machui & 160 & $5(3.1)$ & & & 98 & $8(8.2)$ & 45 & $1(2.2)$ & $-0.9 \%(0.7509)$ \\
\hline Kitogani & & & 106 & $2(1.9)$ & 77 & $4(5.2)$ & 70 & $5(7.1)$ & $5.2 \%(0.0808)$ \\
\hline Bwejuu & & & 75 & $0(0.0)$ & 101 & $0(0.0)$ & 65 & $1(1.5)$ & $1.5 \%(0.4643)$ \\
\hline Mzambarauni & & & 144 & $79(54.9)$ & & & 85 & $22(25.9)$ & $-29.0 \%(<0.0001)$ \\
\hline Kinowe & & & 158 & $116(73.4)$ & & & 101 & $46(45.5)$ & $-27.9 \%(<0.0001)$ \\
\hline Vitongoji & & & 119 & $63(52.9)$ & & & 80 & $31(38.8)$ & $-14.1 \%(0.0493)$ \\
\hline Ngwachani & & & 167 & $156(93.4)$ & & & 104 & $55(52.9)$ & $-40.5 \%(<0.0001)$ \\
\hline Total & & reened (Feb & and Jul 200 & 7): 1,279; Ca & ses: 461 (36 & 6.0) & 767 & $173(22.6)$ & $-13.4 \%(<0.0001)$ \\
\hline \multicolumn{10}{|c|}{ Trichuris trichiura infection } \\
\hline \multirow{2}{*}{ School } & 1st visit (F & Feb 2007) & 2nd visit & (Jul 2007) & 3rd visit (F & (Feb 2008) & 4th visit (F & Feb 2009) & Difference between 1st and \\
\hline & Screened & Cases (\%) & Screened & Cases (\%) & Screened & Cases (\%) & Screened & Cases (\%) & 4th screening ( $P$-value) \\
\hline Chaani & 129 & $5(3.9)$ & & & 61 & $9(14.8)$ & 43 & $6(14.0)$ & $10.1 \%(0.0193)$ \\
\hline Kilombero & 105 & $31(29.5)$ & & & 62 & $15(24.2)$ & 114 & $8(7.0)$ & $-22.5 \%(<0.0001)$ \\
\hline Ghana & 121 & $26(21.5)$ & & & 88 & $9(10.2)$ & 60 & $8(13.3)$ & $-8.2 \%(0.1861)$ \\
\hline Machui & 160 & $12(7.5)$ & & & 99 & $12(12.1)$ & 45 & $6(13.3)$ & $5.8 \%(0.2219)$ \\
\hline Kitogani & & & 105 & $24(22.9)$ & 77 & $6(7.8)$ & 70 & $5(7.1)$ & $-15.8 \%(0.0062)$ \\
\hline Bwejuu & & & 75 & $0(0.0)$ & 100 & $12(12.0)$ & 65 & $6(9.2)$ & $9.2 \%(0.0088)$ \\
\hline Mzambarauni & & & 144 & $102(70.8)$ & & & 84 & 73 (86.9) & $16.1 \%(0.0056)$ \\
\hline Kinowe & & & 158 & $127(80.4)$ & & & 101 & $86(85.1)$ & $4.7 \%(0.3274)$ \\
\hline Vitongoji & & & 119 & $95(79.8)$ & & & 80 & $74(92.5)$ & $12.7 \%(0.0143)$ \\
\hline Ngwachani & & & 167 & $152(91.0)$ & & & 104 & $85(81.7)$ & $-9.3 \%(0.0248)$ \\
\hline Total & & reened (Feb & and Jul 200 & 7): 1,283; Ca & ses: 574 (44 & 4.7) & 766 & $357(46.6)$ & $+1.9 \%(0.4116)$ \\
\hline Hookworm infe & & & & & & & & & \\
\hline Crhont & 1st visit (F & Feb 2007) & 2nd visit & (Jul 2007) & 3rd visit (F & (Feb 2008) & 4th visit (F & Feb 2009) & Difference between 1st and \\
\hline Scritool & Screened & Cases (\%) & Screened & Cases (\%) & Screened & Cases (\%) & Screened & Cases (\%) & \\
\hline Chaani & 129 & $2(1.6)$ & & & 61 & $0(0.0)$ & 43 & $0(0.0)$ & $-1.6 \%(1)$ \\
\hline Kilombero & 106 & $15(14.2)$ & & & 62 & $7(11.3)$ & 114 & $5(4.4)$ & $-9.8 \%(0.0118)$ \\
\hline Ghana & 121 & $15(12.4)$ & & & 88 & $2(2.3)$ & 60 & $2(3.3)$ & $-9.1 \%(0.0491)$ \\
\hline Machui & 160 & $9(5.6)$ & & & 99 & $8(8.1)$ & 45 & $1(2.2)$ & $-3.4 \%(0.3492)$ \\
\hline Kitogani & & & 106 & $1(0.9)$ & 77 & $1(1.3)$ & 70 & $3(4.3)$ & $3.4 \%(0.1453)$ \\
\hline Bwejuu & & & 75 & $4(5.3)$ & 101 & $2(2.0)$ & 65 & $0(0.0)$ & $-5.3 \%(0.1235)$ \\
\hline Mzambarauni & & & 144 & $78(54.2)$ & & & 84 & $52(61.9)$ & $7.7 \%(0.2549)$ \\
\hline Kinowe & & & 158 & $108(68.4)$ & & & 100 & $65(65.0)$ & $-3.4 \%(0.5765)$ \\
\hline Vitongoji & & & 119 & $54(45.4)$ & & & 80 & 55 (68.8) & $23.4 \%(0.0012)$ \\
\hline Ngwachani & & & 167 & $106(63.5)$ & & & 104 & 43 (41.3) & $-22.2 \%(0.0004)$ \\
\hline Total & & reened (Feb & and Jul 200 & 7): 1,285; Ce & ses: 392 (30 & $0.5)$ & 765 & $226(29.5)$ & $-1 \%(0.6457)$ \\
\hline
\end{tabular}

${ }^{*} P$-values were calculated using chi-square or Fisher exact tests. 


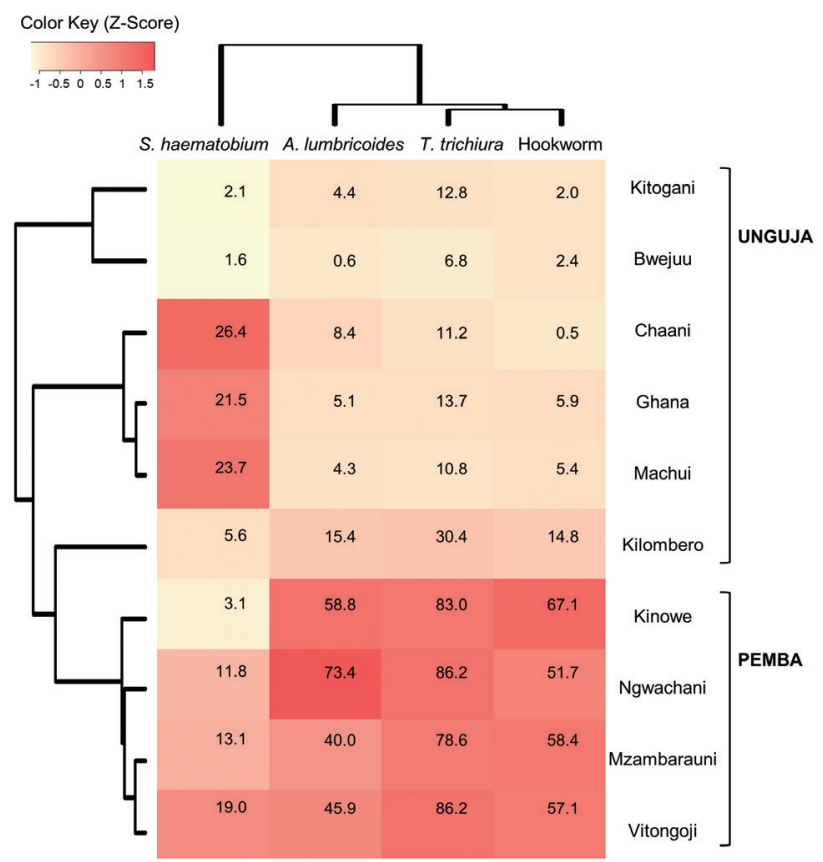

Fig. 2. Heatmap showing the prevalence of soil-transmitted helminth infections and schistosomiasis in children from 10 schools in Zanzibar, Tanzania (2007-2009). Values in the cells represent the 3-year average prevalence of each parasitic infection in each school. The colors on the heatmap are based on z-scores calculated using relative prevalence of parasites infections (Z-score= [actual prevalence mean prevalence along the 10 schools] /standard deviation).

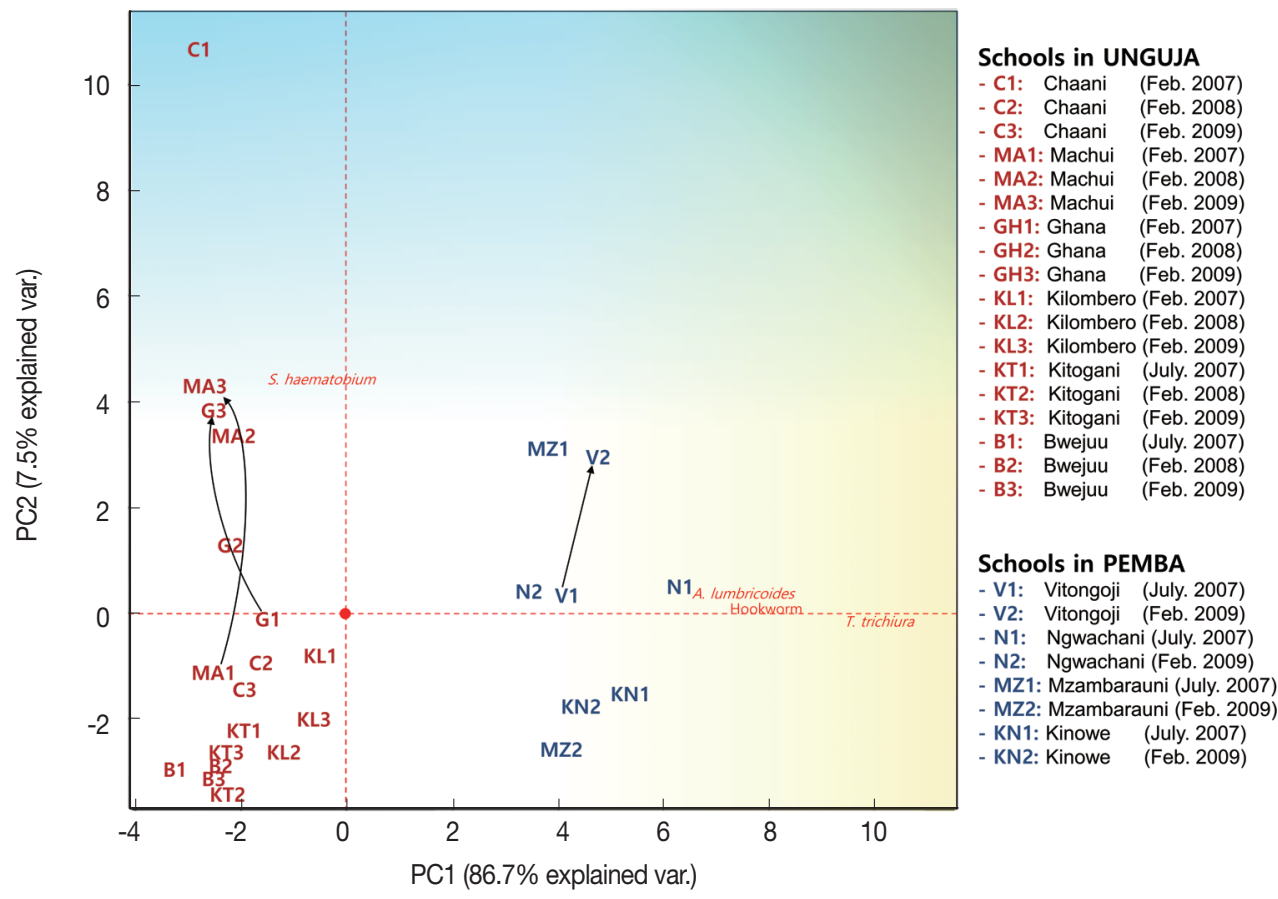

Fig. 3. Principal Component Analysis plot showing helminth infections and schistosomiasis prevalence in children from 10 schools in Zanzibar, Tanzania (2007-2009). Prevalence of Ascaris lumbricoides, Trichuris trichiura, hookworm, and Schistosoma haematobium infection in 10 schools for three years was subjected to principal component analysis and the first 2 principal components, PC1 and PC2, were plotted. PC1 explained the $86.7 \%$ of the total variability found, and PC2 explained the $7.5 \%$ of the total variability. Black arrows indicate schools with increased parasitic infection status. 
Table 3. Association analysis of variables related to parasitic infection

\begin{tabular}{|c|c|c|c|c|c|}
\hline & $\begin{array}{c}\text { Schistosoma } \\
\text { haematobium } \\
\text { cases/screened (\%) }\end{array}$ & $\begin{array}{c}\text { Ascaris } \\
\text { lumbricoides } \\
\text { cases/screened (\%) }\end{array}$ & $\begin{array}{c}\text { Trichuris } \\
\text { trichiura } \\
\text { cases/screened (\%) }\end{array}$ & $\begin{array}{c}\text { Hookworm } \\
\text { cases/screened (\%) }\end{array}$ & $\begin{array}{c}\text { Anemia } \\
\text { cases/screened (\%) }\end{array}$ \\
\hline $\begin{array}{l}\text { Island } \\
\text { Unguja } \\
\text { Pemba } \\
\text { Total }\end{array}$ & $\begin{array}{c}P=0.002^{*} \\
87 / 653(13.3) \\
27 / 379(7.1) \\
114 / 1,032(11.0)\end{array}$ & $\begin{array}{c}P<0.001 \\
19 / 397(4.8) \\
124 / 292(42.5) \\
143 / 689(20.8)\end{array}$ & $\begin{array}{c}P<0.001 \\
39 / 397(9.8) \\
246 / 291(84.5) \\
285 / 688(41.4)\end{array}$ & $\begin{array}{c}P<0.001 \\
11 / 397(2.8) \\
161 / 290(55.5) \\
172 / 687(25.0)\end{array}$ & $\begin{array}{c}P<0.001 \\
162 / 479(33.8) \\
106 / 223(47.5) \\
268 / 702(38.2)\end{array}$ \\
\hline $\begin{array}{l}\text { Sex } \\
\text { Male } \\
\text { Female } \\
\text { Total }\end{array}$ & $\begin{array}{c}P<0.001 \\
69 / 412(16.7) \\
44 / 619(7.1) \\
113 / 1,031(11.0)\end{array}$ & $\begin{array}{c}P=0.916 \\
54 / 259(20.8) \\
88 / 429(20.5) \\
142 / 688(20.6)\end{array}$ & $\begin{array}{c}P=0.419 \\
102 / 259(39.4) \\
182 / 428(42.5) \\
284 / 687(41.3)\end{array}$ & $\begin{array}{c}P=0.777 \\
63 / 259(24.3) \\
108 / 427(25.3) \\
171 / 686(24.9)\end{array}$ & $\begin{array}{c}P=0.010 \\
124 / 282(44.0) \\
144 / 420(34.3) \\
268 / 702(38.2)\end{array}$ \\
\hline $\begin{array}{l}\text { Anemia } \\
\text { Yes } \\
\text { No } \\
\text { Total }\end{array}$ & $\begin{array}{c}P=0.620 \\
30 / 263(11.4) \\
44 / 431(10.2) \\
74 / 694(10.7)\end{array}$ & $\begin{array}{c}P=0.017 \\
45 / 195(23.1) \\
45 / 306(14.7) \\
90 / 501(18.0)\end{array}$ & $\begin{array}{c}P=0.051 \\
80 / 195(41.0) \\
99 / 305(32.5) \\
179 / 500(35.8)\end{array}$ & $\begin{array}{c}P=0.077 \\
52 / 194(26.8) \\
61 / 305(20.0) \\
113 / 499(22.6)\end{array}$ & $\begin{array}{l}- \\
- \\
- \\
-\end{array}$ \\
\hline $\begin{array}{l}\text { Hematuria } \\
\text { Yes } \\
\text { No } \\
\text { Total }\end{array}$ & $\begin{array}{c}P<0.001 \\
90 / 149(60.4) \\
18 / 762(2.4) \\
108 / 911(11.9)\end{array}$ & $\begin{array}{c}P=0.404 \\
22 / 80(27.5) \\
113 / 487(23.2) \\
135 / 567(23.8)\end{array}$ & $\begin{array}{c}P=0.110 \\
45 / 80(56.3) \\
227 / 487(46.6) \\
272 / 567(48.0)\end{array}$ & $\begin{array}{c}P=0.330 \\
27 / 80(33.8) \\
138 / 486(28.4) \\
165 / 566(29.2)\end{array}$ & $\begin{array}{c}P=0.040 \\
46 / 93(49.5) \\
200 / 524(38.2) \\
246 / 617(39.9)\end{array}$ \\
\hline $\begin{array}{l}\text { Previous S. haematobium infection } \\
\text { Yes } \\
\text { No } \\
\text { Total }\end{array}$ & $\begin{array}{c}P<0.001 \\
53 / 159(33.3) \\
61 / 838(7.3) \\
114 / 996(11.4)\end{array}$ & $\begin{array}{c}P=0.117 \\
12 / 88(13.6) \\
121 / 582(20.8) \\
133 / 670(19.9)\end{array}$ & $\begin{array}{c}P=0.210 \\
30 / 88(34.1) \\
239 / 581(41.1) \\
269 / 669(40.2)\end{array}$ & $\begin{array}{c}P=0.356 \\
18 / 88(20.5) \\
145 / 580(25.0) \\
163 / 668(24.4)\end{array}$ & $\begin{array}{l}- \\
- \\
- \\
-\end{array}$ \\
\hline $\begin{array}{l}\text { Previous A. lumbricoides infection } \\
\text { Yes } \\
\text { No } \\
\text { Total }\end{array}$ & $\begin{array}{c}P=0.231 \\
30 / 293(10.2) \\
74 / 567(13.1) \\
104 / 860(12.1)\end{array}$ & $\begin{array}{c}P<0.001 \\
92 / 238(38.7) \\
26 / 355(7.3) \\
118 / 593(19.9)\end{array}$ & $\begin{aligned} P<0.001 \\
172 / 238(72.3) \\
71 / 354(20.1) \\
243 / 592(41.0)\end{aligned}$ & $\begin{aligned} P<0.001 \\
103 / 237(43.5) \\
41 / 354(11.6) \\
144 / 591(24.4)\end{aligned}$ & $\begin{array}{l}- \\
- \\
-\end{array}$ \\
\hline $\begin{array}{l}\text { Previous T. trichiura infection } \\
\text { Yes } \\
\text { No } \\
\text { Total }\end{array}$ & $\begin{array}{c}P=0.498 \\
41 / 366(11.2) \\
63 / 495(12.7) \\
104 / 861(12.1)\end{array}$ & $\begin{array}{c}P<0.001 \\
95 / 277(34.3) \\
23 / 314(7.3) \\
118 / 591(20.0)\end{array}$ & $\begin{aligned} & P<0.001 \\
& 181 / 277(65.3) \\
& 62 / 313(19.8) \\
& 243 / 590(41.2)\end{aligned}$ & $\begin{array}{c}P<0.001 \\
110 / 276(39.9) \\
34 / 313(10.9) \\
144 / 589(24.4)\end{array}$ & $\begin{array}{l}- \\
- \\
-\end{array}$ \\
\hline $\begin{array}{l}\text { Previous hookworm infection } \\
\text { Yes } \\
\text { No } \\
\text { Total }\end{array}$ & $\begin{array}{c}P=0.170 \\
23 / 240(9.5) \\
81 / 624(13.0) \\
104 / 864(12.0)\end{array}$ & $\begin{array}{c}P<0.001 \\
71 / 191(37.2) \\
49 / 403(12.2) \\
120 / 594(20.2)\end{array}$ & $\begin{array}{c}P<0.001 \\
143 / 191(74.9) \\
102 / 402(25.4) \\
245 / 593(41.3)\end{array}$ & $\begin{array}{c}P<0.001 \\
98 / 190(51.6) \\
47 / 402(11.7) \\
145 / 592(24.5)\end{array}$ & $\begin{array}{l}- \\
- \\
-\end{array}$ \\
\hline
\end{tabular}

${ }^{*} P$-values were calculated using chi-square or Fisher exact tests.

haematobium infection prevalence were at right angles, which explains the mode of infection of STHs and S. haematobium are different (or independent). In contrast, the vectors of $A$. lumbricoides, hookworm, and T. trichiura infection prevalence were similar (to the right), which suggested that STH infections are closely interrelated (or dependent).

In general, the Unguja schools were less infected by STHs (left side of PCA plot) than the Pemba schools (right side of PCA plot), and the parasitic infection status of most schools was generally improved or maintained during the survey period. However, infection status worsened in the Machui, Ghana, and Vitongoji schools, and such an increase could mainly be attributed to remarkable increases in the prevalence of $S$. haematobium infection (black arrows in Fig. 3 and Table 2).

Meanwhile, the Unguja schools were more infected by $S$. haematobium than the Pemba schools $(P=0.002)$, and boys were more infected than girls $(P<0.001)$, probably because the boys spent more time playing in the water (Table 3 ).

In regard to hemoglobin concentrations and urine analysis, chi-square analysis indicated that $S$. haematobium infection was associated with hematuria $(P<0.001)$ but not with anemia $(P=0.620)$, whereas STH infection was associated with anemia (Table 3). However, since anemia was more prevalent in the Pemba schools, careful interpretation is needed. Interestingly, the probability of parasitic infection seemed to be influenced by previous parasitic infection (Table 3).

Multiple logistic regression analysis indicated that children in Unguja possessed $~ 1.7$-fold greater risk of $S$. haematobium infection than children in Pemba (Table 4), and the infection rate of boys was significantly greater than that of girls, with an odds ratio (OR) of 2.449. In addition, children diagnosed with S. haematobium infection in the previous visit had 5.5-fold 
Table 4. Multiple logistic regression for risk factor assessment for parasitic infections in the children in Zanzibar in 2009. The information on the previous parasitic infections was derived from the egg examination in 2007 or 2008

\begin{tabular}{|c|c|c|c|c|}
\hline & $\begin{array}{c}\text { Schistosoma } \\
\text { haematobium } \\
\text { odds ratio }(95 \% \mathrm{Cl})\end{array}$ & $\begin{array}{c}\text { Ascaris } \\
\text { lumbricoides } \\
\text { odds ratio }(95 \% \mathrm{Cl})\end{array}$ & $\begin{array}{c}\text { Trichuris } \\
\text { trichiura } \\
\text { odds ratio }(95 \% \mathrm{Cl})\end{array}$ & $\begin{array}{c}\text { Hookworm } \\
\text { odds ratio }(95 \% \mathrm{Cl})\end{array}$ \\
\hline $\begin{array}{l}\text { Island } \\
\text { Unguja } \\
\text { Pemba } \\
P \text {-value }\end{array}$ & $\begin{array}{l}1.705(1.053-2.763) \\
\quad \text { Reference } \\
{ }^{*} P=0.030\end{array}$ & $\begin{array}{l}0.100(0.051-0.198) \\
\text { Reference } \\
{ }^{\star} P<0.001\end{array}$ & $\begin{array}{c}0.025(0.014-0.045) \\
\text { Reference } \\
{ }^{*} P<0.001\end{array}$ & $\begin{array}{c}0.029(0.014-0.062) \\
\text { Reference } \\
{ }^{\star} P<0.001\end{array}$ \\
\hline $\begin{array}{l}\text { Sex } \\
\text { Male } \\
\text { Female } \\
P \text {-value }\end{array}$ & $\begin{array}{l}2.449(1.608-3.731) \\
\quad \text { Reference } \\
{ }^{*} P<0.001\end{array}$ & $\begin{array}{c}1.314(0.820-2.105) \\
\text { Reference } \\
P=0.256\end{array}$ & $\begin{array}{c}1.064(0.645-1.757) \\
\quad \text { Reference } \\
P=0.808\end{array}$ & $\begin{array}{c}1.179(0.721-1.925) \\
\text { Reference } \\
P=0.512\end{array}$ \\
\hline $\begin{array}{l}\text { Previous S. haematobium infection } \\
\text { Yes } \\
\text { No } \\
P \text {-value }\end{array}$ & $\begin{array}{l}5.557(3.608-8.558) \\
\quad \text { Reference } \\
\quad{ }^{*} P<0.001\end{array}$ & $\begin{array}{l}- \\
-\end{array}$ & - & - \\
\hline $\begin{array}{l}\text { Previous A. lumbricoides infection } \\
\text { Yes } \\
\text { No } \\
P \text {-value }\end{array}$ & - & $\begin{array}{c}2.093(1.157-3.787) \\
\text { Reference } \\
{ }^{*} P=0.015\end{array}$ & - & - \\
\hline $\begin{array}{l}\text { Previous T. trichiura infection } \\
\text { Yes } \\
\text { No } \\
P \text {-value }\end{array}$ & $\begin{array}{l}- \\
-\end{array}$ & - & $\begin{array}{c}1.248(0.694-2.244) \\
\text { Reference } \\
P=0.460\end{array}$ & - \\
\hline $\begin{array}{l}\text { Previous Hookworm infection } \\
\text { Yes } \\
\text { No } \\
P \text {-value }\end{array}$ & - & - & - & $\begin{array}{c}1.819(1.091-3.030) \\
\text { Reference } \\
{ }^{*} P=0.022\end{array}$ \\
\hline
\end{tabular}

${ }^{*} \mathrm{OR}$, odds ratio; $\mathrm{Cl}$, confidence interval.

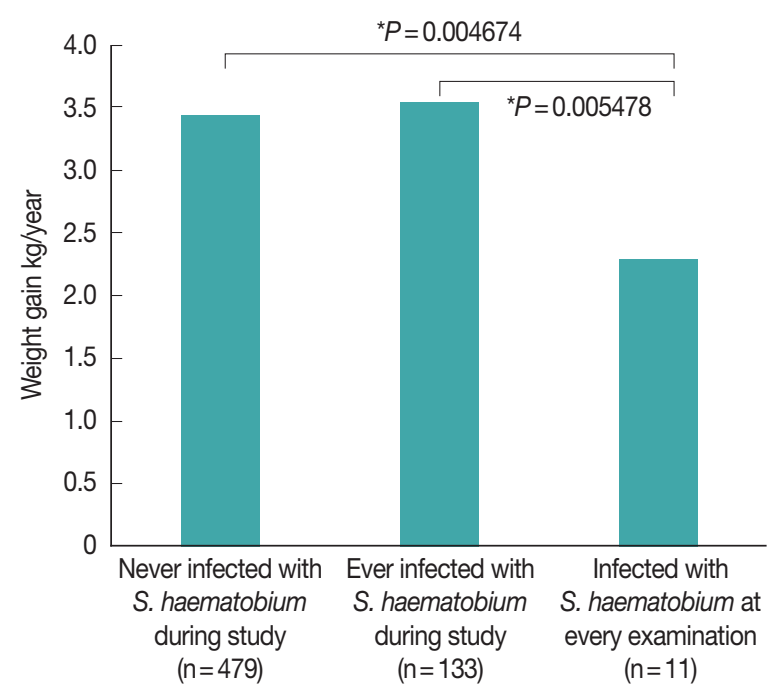

Fig. 4. Effect of Schistosoma haematobium infection on the weight gain of schoolchildren in Zanzibar, Tanzania. Bonferroni corrected $P$-values are shown.

greater risk of present $S$. haematobium infection.

Meanwhile, children in Pemba were 10 to 40 times more likely to present STH infection than children in Unguja, and even though children diagnosed with A. lumbricoides or hookworm infection were more likely ( $\sim 2$-fold) to exhibit re-infection at future visits, the risk was much lower than that observed for $S$. haematobium re-infection $(\mathrm{OR}=5.557)$. In contrast, T. trichiura infection did not have a significant effect on future infection $T$. trichiura status $(P=0.460)$. Together, these results demonstrate that $S$. haematobium is more capable of maintaining or re-establishing infection status than are STHs after MDA.

The annual weight gain of children who presented with $S$. haematobium infection at any point during the survey period (3.54 kg/year) was statistically similar to that of children who did not (3.47 kg/year; Fig. 4). However, the annual weight gain of children who presented with $S$. haematobium at every examination $(2.28 \mathrm{~kg} /$ year $)$ was significantly lower than that of children who had either never presented with $S$. haematobium infection $(P=0.00468)$ and who had only presented with $S$. haematobium infection during a portion of visits $(P=0.00548)$. In contrast, there was no difference in the annual weight gain of children who presented with STH infection at every examination $(3.49 \mathrm{~kg} /$ year) and that of children who never present- 
Table 5. Evaluation of hematuria as a predictor of Schistosoma haematobium infection

\begin{tabular}{|c|c|c|c|c|c|}
\hline \multirow[b]{2}{*}{ All schools in Zanzibar for 3 years } & \multirow[b]{2}{*}{ Egg Positive } & \multirow{2}{*}{$\begin{array}{c}\text { Hematuria Positive } \\
310\end{array}$} & \multirow{2}{*}{$\begin{array}{c}\text { Hematuria Negative } \\
85\end{array}$} & \multicolumn{2}{|c|}{ Combo values } \\
\hline & & & & $\begin{array}{l}\text { Sensitivity } \\
\text { Specificity }\end{array}$ & $\begin{array}{l}78.48 \% \\
92.66 \%\end{array}$ \\
\hline & Egg Negative & 201 & 2,539 & $\begin{array}{l}\text { PPV } \\
\text { NPV }\end{array}$ & $\begin{array}{l}60.67 \% \\
96.76 \%\end{array}$ \\
\hline \multirow[t]{2}{*}{ Machui school for 3 years } & Egg Positive & 77 & 27 & $\begin{array}{l}\text { Sensitivity } \\
\text { Specificity }\end{array}$ & $\begin{array}{l}74.04 \% \\
96.71 \%\end{array}$ \\
\hline & Egg Negative & 10 & 294 & $\begin{array}{l}\text { PPV } \\
\text { NPV }\end{array}$ & $\begin{array}{l}88.51 \% \\
91.59 \%\end{array}$ \\
\hline
\end{tabular}

PPV, positive predictive value; NPV, negative predictive value.

ed with STH infection (3.14 kg/year, $P=0.2271)$.

The sensitivity, specificity, positive predictive value (PPV), and negative predictive value (NPV) were calculated using the values in Table 5. PPV indicates the rate of cases that were infected with $S$. haematobium (egg positive) when hematuria was present; NPV is the rate of cases that were not infected with $S$. haematobium when hematuria was absent. Across the 10 schools, the sensitivity, specificity, PPV, and NPV of hematuria for $S$. haematobium infection were 78.48, 92.66, 60.67, and $96.76 \%$, respectively; in the Machui school, which had the highest $S$. haematobium infection rate, the PPV was $88.51 \%$.

\section{DISCUSSION}

In the present study, overall infection status was assessed, and MDA was applied to a cohort of schoolchildren from schools across Zanzibar. Heatmap (Fig. 2) and multiple regression (Table 4) analyses indicated that elementary schools in Pemba had a much higher prevalence of STH infections than those in Unguja. The different STH infection patterns of these 2 islands (Pemba and Unguja) indicate that the control of STH infection in Unguja has been consistently more effective than that in Pemba, which is generally less developed.

The present study also revealed that the prevalence of $S$. haematobium infection varies markedly by region. In Unguja, the Chaani, Ghana, and Machui schools possessed high rates of $S$. haematobium infection, whereas the Kitogani and Bwejuu schools, which were located far from the streams that harbor the intermediate host organism, possessed very low infection rates. These findings agree with those of French at al., who reported that urinary schistosomiasis was not endemic to either the Kitogani or Bwejuu [14].

Furthermore, the effectiveness of the MDA program in the present study seems controversial. Even though the infection rates of the Chaani and Mzambarauni schools were reduced by 55.1 and $21.1 \%$ (Table 2), respectively, the overall reduction across all 10 schools was only $1.8 \%$, and even that small reduction was not statistically significant $(P=0.1751)$. Furthermore, the $S$. haematobium infection rates of several schools (Machui, Ghana, and Vitongoji) actually exhibited significant increases (Table 2). In addition, of the 3 STHs, MDA was only effective against $A$. lumbricoides. The rate of $A$. lumbricoides infection across all schools was $36.0 \%$ at the beginning of the project and was reduced to $22.6 \%$ by the last screening $(P<0.0001)$. Therefore, MDA significantly reduced the rate of A. lumbricoides infection, especially in the 4 schools in Pemba. However, there were no significant effects on the overall $T$. trichiura and hookworm infection rates across all 10 schools (Table 2).

The MDA protocol used in the present study was only effective in reducing A. lumbricoides infection, a finding that is supported by previous studies [17]. It is possible that the MDA interval should have been shorter and that the study period should have been longer in order to effectively control STH infections. According to previous studies, MDA programs for reducing STH infection rates should include at least 2 treatments a year for 3 years [18-20]. In addition, chemotherapy for general residents who were not included in the parasitic control programs could reduce re-infection and lower overall infection rates for STHs.

In the present study, S. haematobium was more capable of reinfecting children than STHs (Table 4). Similarly, high rates of schistosome re-infection have been reported previously [21] and could possibly be attributed to the ineffectiveness of praziquantel in killing juvenile schistosomes [22] or the fact that schistosomes are can be rapidly transmitted to snails by urination from only a few infected humans.

According to these results, parasitic control programs that 

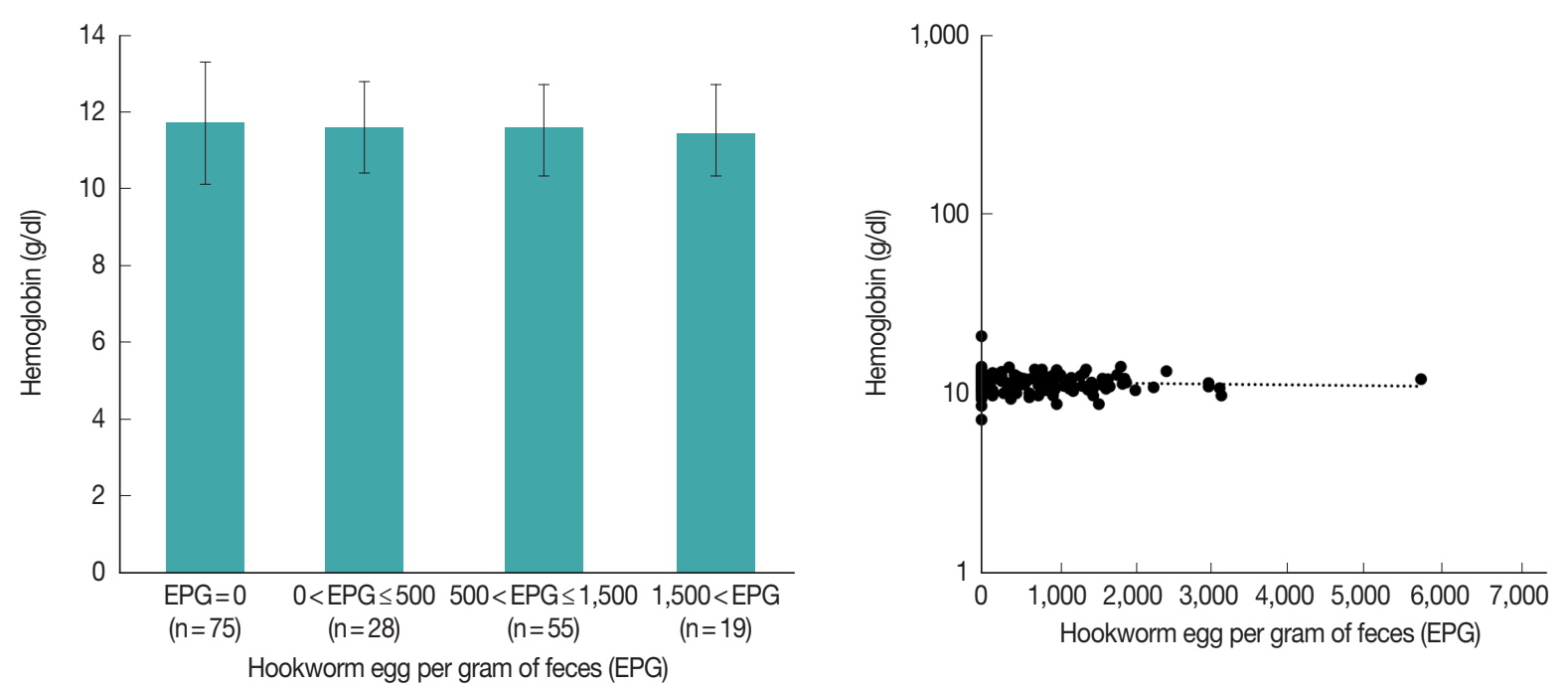

Fig. 5. (A) Hemoglobin level by hookworm EPG grade. The data of the children on the Pemba island in 2009 were used. There were no significant differences among the groups ( $P$-value for one-way ANOVA=0.892). Bars represent the standard error of the mean. $(B)$ Scatter plot and linear regression analysis for hemoglobin concentration and hookworm EPG. The data of the children on the Pemba island in 2009 were used. $P$-value for coefficient was 0.968.

are solely based on chemotherapy are not effective or sustainable for the long-term prevention of re-infections. Instead, there is a need for environmental improvements, such as the development of safe water and sanitation resources and changes in hygiene behavior [23,24]. In addition, residents that are not included in parasitic control programs can function as parasite reservoirs, thereby facilitating continuous transmission [25], and snail control, using molluscicides (e.g., niclosamide), should be employed to interrupt re-infection cycles $[26,27]$.

This study revealed that children who were infected with $S$. haematobium at every visit demonstrated lower annual weight gain than the other groups, whereas children who were cured from the parasitic infection at least once exhibited normal growth (Fig. 4). Therefore, despite the fact that school-based MDA for schistosomiasis appears to be somewhat ineffective owing to the high re-infection rate, it may still be particularly beneficial to the growth of children.

The present study found that hematuria, a key symptom of S. haematobium infection, was useful for diagnosing S. haematobium infection. Its sensitivity, specificity, PPV, and NPV for $S$. haematobium infection were 78.5, 92.7, 60.7, and 96.8\%, respectively, across all schools and even higher if calculated using data from schools with high $S$. haematobium infection rates. Therefore, it is possible that praziquantel could be prescribed on the basis of hematuria alone if it was impossible to perform microscopic egg detection in a highly S. haematobium endemic region, such as Zanzibar.

It is known that hookworm causes anemia, but in this study, hookworm was not significantly associated with anemia (Table 3). For further analysis, we compared hemoglobin levels according to the amount of EPG in Pemba in 2009; however, there was still no difference between the groups (Fig. 5A, Pvalue for ANOVA=0.892). Additionally, in linear regression analysis, hemoglobin and EPG were not correlated (Fig. 5B, Pvalue for coefficient $(b)=0.968)$. In Zanzibar, anemia due to malnutrition in children is a serious problem, which might hide the effect of the hookworm on anemia.

A recent study reported that the prevalence of urogenital schistosomiasis was reduced from $6.1 \%$ to $1.7 \%$ in elementary school children and from $3.9 \%$ to $1.5 \%$ in adults, from 2011 to 2017 in Zanzibar [28]. Another schistosomiasis survey of 24 schools showed that the infection rates in Unguja and Pemba were $8 \%$ and $15 \%$, respectively in 2011 [29].

STH infection has been recognized as a problem for many decades, especially on the Pemba island. In 1997, a survey revealed the prevalence in school-aged children to be $72 \%$ for $A$. lumbricoides, 94\% for T. trichiura, and 95\% for hookworm in Pemba [30]. Additionally, Pemba had more STH infection rates than Unguja in our present study as well, which is probably because the Pemba island is less socioeconomically developed than the Unguja island. 
The inhabitants of one region of Tanzania along the coast of Lake Victoria, in Mwanza, suffer from Schistosoma mansoni infections. The prevalence in children was reported to be approximately 40\% in 2011 [31]. In addition, other regions of Tanzania, such as the Rufiji and Mkuranga districts in the Coast Region, were the endemic regions of both S. mansoni and $S$. haematobium [32]. The prevalence of $S$. mansoni was $2.7 \%$ and $1.6 \%$ and that of $S$. haematobium was $10.5 \%$ and $7.0 \%$ in the Rufiji and Mkuranga districts, respectively.

In conclusion, the 10 primary schools surveyed by the present study exhibited different parasitic infections patterns, and a 3-year MDA program was only partially able to control helminth and schistosome infections, owing to high re-infection rates. In the future, parasite control programs should involve strategically designed MDA schedules and holistic intervention (e.g., sanitation improvement, hygiene behavior changes, and control of intermediated hosts).

\section{ACKNOWLEDGMENTS}

This work was implemented through a Korea-Zanzibar collaborative project on health promotion through parasite control among school children in Zanzibar [2007-2009], which was supported by the Korea Foundation for International Healthcare (KOFIH).

\section{CONFLICT OF INTEREST}

The authors declare no conflict of interest related to this study.

\section{REFERENCES}

1. World Health Organization. Accelerating Work to Overcome the Global Impact of Neglected Tropical Diseases: A Roadmap for Implementation: Executive Summary. Geneva, Switzerland. World Health Organization. 2012.

2. World Health Organization. Investing to Overcome the Global Impact of Neglected Tropical Diseases: Third WHO Report on Neglected Diseases 2015. Geneva, Switzerland. World Health Organization. 2015.

3. Engels D, Savioli L. Reconsidering the underestimated burden caused by neglected tropical diseases. Trends Parasitol 2006; 22: 363-366.

4. Crompton DWT, Savioli L. Handbook of Helminthiasis for Public Health. Boca Raton, USA. CRC Press. 2006.

5. King CH, Dangerfield-Cha M. The unacknowledged impact of chronic schistosomiasis. Chronic Illn 2008; 4: 65-79.

6. Mostafa MH, Sheweita SA, O'Connor PJ. Relationship between schistosomiasis and bladder cancer. Clin Microbiol Rev 1999; 12: 97-111.

7. Molyneux DH, Savioli L, Engels D. Neglected tropical diseases: progress towards addressing the chronic pandemic. Lancet 2017; 389: 312-325

8. World Health Organization. Sustaining the Drive to Overcome the Global Impact of Neglected Tropical Diseases: Second WHO Report on Neglected Tropical Diseases. Geneva, Switzerland. World Health Organization. 2013.

9. Knopp S, Stothard JR, Rollinson D, Mohammed KA, Khamis IS, Marti H, Utzinger J. From morbidity control to transmission control: time to change tactics against helminths on Unguja Island, Zanzibar. Acta Trop 2013; 128: 412-422.

10. Marti H, Haji HJ, Savioli L, Chwaya HM, Mgeni AF, Ameir JS, Hatz C. A comparative trial of a single-dose ivermectin versus three days of albendazole for treatment of Strongyloides stercoralis and other soil-transmitted helminth infections in children. Am J Trop Med Hyg 1996; 55: 477-481.

11. Albonico M, Chwaya HM, Montresor A, Stolfzfus RJ, Tielsch JM, Alawi KS, Savioli L. Parasitic infections in Pemba Island schoolchildren. East Afr Med J 1997; 74: 294-298.

12. Stothard JR, French MD, Khamis IS, Basáñez MG, Rollinson D. The epidemiology and control of urinary schistosomiasis and soil-transmitted helminthiasis in schoolchildren on Unguja island, Zanzibar. Trans R Soc Trop Med Hyg 2009; 103: 1031-1044.

13. Stothard JR, Mgeni AF, Khamis S, Seto E, Ramsan M, Hubbard SJ, Kristensen TK, Rollinson D. New insights into the transmission biology urinary schistosomiasis in Zanzibar. Trans R Soc Trop Med Hyg 2002; 96: 470-475.

14. French MD, Rollinson D, Basanez MG, Mgeni AF, Khamis IS, Stothard JR. School-based control of urinary schistosomiasis on Zanzibar, Tanzania: monitoring micro-haematuria with reagent strips as a rapid urological assessment. J Pediatr Urol 2007; 3: 364-368.

15. Savioli L, Dixon H, Kisumku UM, Mott KE. Control of morbidity due to Schistosoma haematobium on Pemba island; selective population chemotherapy of schoolchildren with haematuria to identify high-risk localities. Trans R Soc Trop Med Hyg 1989; 83: 805-810

16. Lwambo NJ, Savioli L, Kisumku UM, Alawi KS, Bundy DA. The relationship between prevalence of Schistosoma haematobium infection and different morbidity indicators during the course of a control programme on Pemba Island. Trans R Soc Trop Med Hyg 1997; 91: 643-646.

17. Keiser J, Utzinger J. Efficacy of current drugs against soil-transmitted helminth infections: systematic review and meta-analysis. JAMA 2008; 299: 1937-1948.

18. Brooker SJ, Mwandawiro CS, Halliday KE, Njenga SM, Mcharo C, Gichuki PM, Wasunna B, Kihara JH, Njomo D, Alusala D, Chiguzo A, Turner HC, Teti C, Gwayi-Chore C, Nikolay B, Truscott JE, Hollingsworth TD, Balabanova D, Griffiths UK, Freeman 
MC, Allen E, Pullan RL, Anderson RM. Interrupting transmission of soil-transmitted helminths: a study protocol for cluster randomised trials evaluating alternative treatment strategies and delivery systems in Kenya. BMJ Open 2015; 5: e008950.

19. Seo BS, Chai JY. Effect of two-month interval mass chemotherapy on the reinfection of Ascaris lumbricoides in Korea. Korean J Parasitol 1980; 18: 153-163.

20. Seo BS, Cho SY, Chai JY, Hong ST. Comparative efficacy of interval mass treatment on Ascaris lumbricoides infection in Korea. Korean J Parasitol 1980; 18: 145-151.

21. Mwandawiro C, Okoyo C, Kihara J, Simiyu E, Kepha S, Campbell SJ, Freeman MC, Brooker SJ, Njenga SM. Results of a national school-based deworming programme on soil-transmitted helminths infections and schistosomiasis in Kenya: 2012-2017. Parasit Vectors 2019; 12: 76.

22. Gray DJ, McManus DP, Li Y, Williams GM, Bergquist R, Ross AG. Schistosomiasis elimination: lessons from the past guide the future. Lancet Infect Dis 2010; 10: 733-736.

23. Freeman MC, Clasen T, Brooker SJ, Akoko DO, Rheingans R. The impact of a school-based hygiene, water quality and sanitation intervention on soil-transmitted helminth reinfection: a clusterrandomized trial. Am J Trop Med Hyg 2013; 89: 875-883.

24. Okoyo C, Nikolay B, Kihara J, Simiyu E, Garn JV, Freeman MC, Mwanje MT, Mukoko DA, Brooker SJ, Pullan RL, Njenga SM, Mwandawiro CS. Monitoring the impact of a national school based deworming programme on soil-transmitted helminths in Kenya: the first three years, 2012-2014. Parasit Vectors 2016; 9: 408.

25. Dana D, Mekonnen Z, Emana D, Ayana M, Getachew M, Workneh N, Vercruysse J, Levecke B. Prevalence and intensity of soiltransmitted helminth infections among pre-school age children in 12 kindergartens in Jimma Town, southwest Ethiopia. Trans
R Soc Trop Med Hyg 2014; 109: 225-227.

26. Coelho P, Caldeira RL. Critical analysis of molluscicide application in schistosomiasis control programs. Infect Dis Poverty 2016; 5: 4-9.

27. Knopp S, Mohammed KA, Ali SM, Khamis IS, Ame SM, Albonico M, Gouvras A, Fenwick A, Savioli L, Colley DG, Utzinger J, Person B, Rollinson D. Study and implementation of urogenital schistosomiasis elimination in Zanzibar (Unguja and Pemba islands) using an integrated multidisciplinary approach. BMC Public Health 2012; 12: 930.

28. Knopp S, Ame SM, Person B, Hattendorf J, Rabone M, Juma S, Muhsin J, Khamis IS, Hollenberg E, Mohammed KA, Kabole F, Ali SM, Rollinson D. A 5-Year intervention study on elimination of urogenital schistosomiasis in Zanzibar: Parasitological results of annual cross-sectional surveys. PLoS Negl Trop Dis 2019; 13: e0007268.

29. Knopp S, Person B, Ame SM, Mohammed KA, Ali SM, Khamis IS, Rabone M, Allan F, Gouvras A, Blair L, Fenwick A, Utzinger J, Rollinson D. Elimination of schistosomiasis transmission in Zanzibar: baseline findings before the onset of a randomized intervention trial. PLoS Negl Trop Dis 2013; 7: e2474.

30. Albonico M, Chwaya HM, Montresor A, Stolfzfus RJ, Tielsch JM, Alawi KS, Savioli L. Parasitic infections in Pemba Island school children. East Afr Med J 1997; 74: 294-298.

31. Olsen A, Kinung'hi S, Magnussen P. Schistosoma mansoni infection along the coast of Lake Victoria in Mwanza region, Tanzania. Am J Trop Med Hyg 2015; 92: 1240-1244.

32. Madon S, Malecela MN, Mashoto K, Donohue R, Mubyazi G, Michael E. The role of community participation for sustainable integrated neglected tropical diseases and water, sanitation and hygiene intervention programs: a pilot project in Tanzania. Soc Sci Med 2018; 202: 28-37. 
